'Programa de Doctorado en Biología y Ecología Aplicada. Coquimbo, Chile.

${ }^{2}$ Departamento de Salud Pública, Facultad de Medicina, Universidad Católica del Norte. Coquimbo, Chile.

${ }^{3}$ Departamento de Biología Marina, Universidad Católica de Norte. Coquimbo, Chile.

${ }^{4}$ Instituto de Salud Pública de Chile.

${ }^{5}$ Facultad de Biología, Universidad de Barcelona. España.

Los autores declaran no tener conflictos de interés. Trabajo no recibió financiamiento.

Recibido el 31 de julio de 2017, aceptado el 11 de abril de 2018.

Correspondencia a: Dra. (c) Isabel Valdés Aravena Programa de Doctorado en Biología y Ecología Aplicada, Universidad Católica del Norte.

Larrondo 1281, Casilla 117 , Coquimbo, Chile. Tel: +56 (51) 2205963. ipvaldes@uc.c

\section{Medicina traslacional e innovación en salud: mecanismos y perspectivas}

\author{
ISABEL P. VALDÉS ${ }^{1}$, MURIEL RAMÍREZ-SANTANA², \\ ANDREA BASAGOITÍA ${ }^{4}$, XAVIER TESTAR ${ }^{5}$, JULIO A. VÁSQUEZ ${ }^{3}$

\section{Translational medicine and innovation in health: mechanisms and perspectives}

Many new discoveries in Life Sciences cannot be translated into products, services or new applications to improve human health. Translational medicine, defined as "from bench to bedside", refers to the transfer of results or new knowledge achieved in the laboratory into health innovation. We aim to review the state of art of translational medicine, its relationship with innovation processes and the different perspectives to consider. Finally, we contextualize the situation of Research and Development $(R \& D)$ in Chile and the main issues of the biotechnology market in the country.

(Rev Med Chile 2018; 146: 890-898)

Key words: Chile; Technology Transfer; Translational Medical Research.
$\mathrm{E}$ n las últimas décadas, avances significativos en las ciencias biológicas, han generado mayor conocimiento de los mecanismos implicados en las enfermedades humanas ${ }^{1}$. Sin embargo, menos de 5\% de todos los descubrimientos en el área de ciencias de la vida, han producido nuevos medicamentos, equipos para tratamientos, cambios en la práctica clínica o en métodos de diagnóstico ${ }^{2}$. El alto costo de la investigación biomédica en universidades y centros de investigación ${ }^{3}$, y la necesidad de mejorar la calidad de vida de los pacientes, han influido en que los tomadores de decisiones quieran poner especial atención en las brechas que dificultan transformar el conocimiento en herramientas que mejoren la salud humana ${ }^{4}$.

En la mayoría de los países que integran la Organización para la Cooperación y el Desarrollo Económico (OCDE), existe preocupación por aumentar la traslación de los resultados de investigación a la práctica clínica, a la industria biomédica, a los pacientes y finalmente a la salud pública. Chile, no está ajeno a esta problemática.
Un ejemplo de ello, es la escasa contribución bibliográfica de los trabajos de investigación científica nacional, financiados por la Comisión Nacional de Investigación Científica y Tecnológica (CONICYT), en la elaboración de guías clínicas para las ochenta patologías del régimen de garantías en salud (GES) $)^{5}$.

Para acelerar la propagación de nuevas tecnologías o aplicaciones médicas a partir de los resultados científicos, es fundamental conocer las características y los elementos involucrados en el proceso de innovación en salud. Esta revisión contextualiza el concepto de medicina traslacional, y los diversos componentes del proceso de innovación en salud. Pretende generar en el lector, una visión global e integradora de la investigación traslacional, los tipos de innovación y las perspectivas desde las cuales se puede abordar la innovación en salud. Finalmente, se realiza una breve descripción de la "Investigación y Desarrollo" (I+D) en Chile, el mercado biotecnológico y las estrategias para la transferencia de los resultados desde las universidades. 


\section{Medicina traslacional}

El concepto de "medicina traslacional", surge en la década del ' $90^{6,7}$, creciendo exponencialmente en publicaciones científicas durante la década recién pasada ${ }^{8}$. En el transcurso de 2015, hubo 7.000 citaciones en la base de datos PubMed con alguna de las palabras clave: "medicina traslacional", "ciencia traslacional" o "investigación traslacional". La traslación de la investigación biomédica implica transformar los resultados obtenidos en el laboratorio, en avances para la salud de los pacientes ${ }^{10}$. Para esto, se requiere que las partes interesadas tomen conciencia y usen la evidencia proveniente de las investigaciones para informar al sector salud y a los tomadores de decisiones ${ }^{11}$.

Los modelos de investigación traslacional han ido adquiriendo mayor complejidad durante los últimos años, desde un modelo de dos bloques el año 2003, a los más recientes de cuatro etapas ${ }^{12,13}$. En el modelo de dos bloques, el primero (T1), incorpora la transferencia de resultados de laboratorio al desarrollo de métodos de diagnóstico, terapia, prevención y primera prueba en humanos; y el segundo bloque (T2), considera la traslación de los resultados de estudios clínicos a la práctica clínica y la toma de decisiones en salud ${ }^{14,15}$. Este modelo ha sido subdividido en tres y cuatro fases, cuyos principales componentes son: investigación básica, investigación clínica, investigación basada en la práctica, y medición de los impactos en salud ${ }^{15}$. El modelo de traslación más reciente plantea las " $4 \mathrm{~T}$ " en investigación traslacional. La investigación traslacional T1 es identificada como el paso desde la investigación básica a una potencial aplicación clínica. La T2 incorpora estudios de eficacia que muestran la conexión entre las potenciales aplicaciones clínicas y guías basadas en evidencia. La T3, considera la traslación de las recomendaciones y guías a la práctica clínica. Finalmente, la T4 consiste en la evaluación de los resultados en la comunidad o población y su análisis a nivel de salud pública ${ }^{12,16}$.

Los ensayos clínicos permiten conocer el comportamiento de un nuevo producto farmacéutico y su eficacia en el tratamiento de una determinada enfermedad. El Departamento Agencia Nacional de Medicamentos del Instituto de Salud Pública de Chile controla el uso de los compuestos en ensayos clínicos en el país; regulado por la Norma Técnica $N^{\circ} 57$ de 2001. En la Tabla 1, se menciona la descripción de cada una de las fases que requiere este tipo de estudios ${ }^{17}$.

Harvard Catalyst, del área Clínica y del Centro de Ciencia Traslacional de la Universidad de Harvard, emplea un modelo en el que define la investigación traslacional como un espectro de actividades donde los descubrimientos biomédicos pueden generar mejoras en la salud humana, $y$ utiliza las etapas T1, T2, T3 y T4 (Figura 1). La T1, ocurre posterior a la investigación básica y aplicada y se realizan ensayos clínicos fase 1 , donde se utiliza una prueba de concepto de lo desarrollado en laboratorio. La T2, incluye estudios clínicos fase 2 y fase 3 y las guías que permiten la aprobación por parte de la agencia de Administración de Alimentos y Medicamentos del Gobierno de Estados Unidos (FDA). En la T3, el conocimiento es trasladado a la práctica y se realizan estudios clínicos

Tabla 1. Fases de los ensayos clínicos para un nuevo fármaco

\begin{tabular}{|c|c|}
\hline $\begin{array}{l}\text { Fases de los ensayos } \\
\text { clínicos (farmacológico) }\end{array}$ & Descripción \\
\hline Fase I & $\begin{array}{l}\text { Se realiza en un número acotado de voluntarios sanos; } 20-100 \text { voluntarios. Se busca deter- } \\
\text { minar la dosis más baja de acción y la más alta que se puede administrar sin ser perjudicial. } \\
\text { Además, analiza la absorción, distribución, metabolismo y eliminación del fármaco }\end{array}$ \\
\hline Fase II & $\begin{array}{c}\text { Se pretende establecer una prueba de eficacia en cientos de pacientes. Se evalúan efectos } \\
\text { secundarios, dosis y duración del tratamiento }\end{array}$ \\
\hline Fase III & Son ensayos en miles de pacientes. Duran entre 1 y 4 años \\
Fase IV & Son estudios posteriores a la comercialización del producto. Busca verificar la efectividad de \\
& la terapia, detectar nuevas reacciones adversas y/o comprobar la frecuencia de las ya descritas \\
\hline
\end{tabular}

Elaboración propia con información del Instituto de Salud Pública de Chile. 


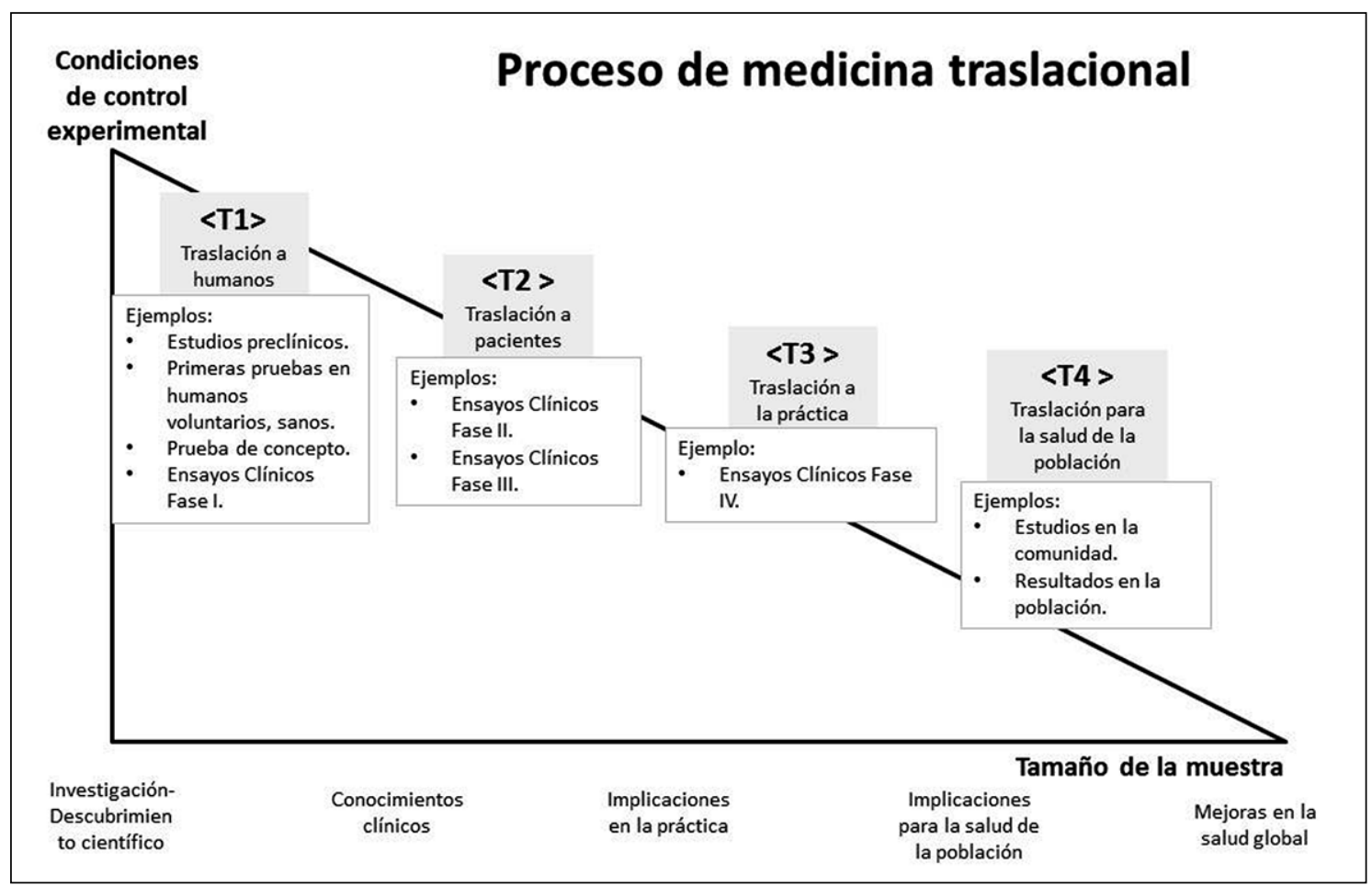

Figura 1. Etapas del Proceso de Medicina Traslacional (T1, T2, T3, T4), partiendo desde un resultado de investigación relevante; adaptado de Harvard Catalyst, 2017. Se observa que las condiciones de control experimental van disminuyendo, mientras el tamaño de la muestra aumenta a medida que transcurre el tiempo de la actividad traslacional.

fase 4. Finalmente, la T4 se enfoca en investigar los resultados y beneficios de la nueva intervención en la sociedad ${ }^{16,18}$.

El Instituto Nacional de Salud de los Estados Unidos (NIH, según sus siglas en inglés) presentó un mapa de ruta para la investigación biomédica, cuyo enfoque está en las brechas críticas para que los descubrimientos científicos generen mejoras en el sector de la salud ${ }^{8}$. Uno de los aspectos más preocupantes a considerar, es el tiempo que se requiere para que un resultado de laboratorio llegue a la práctica clínica, estimándose en 17 años ${ }^{19,20}$. Además, en ese tiempo, los descubrimientos científicos deben conseguir cruzar satisfactoriamente el "valle de la muerte" para cada una de las etapas de la investigación traslacional ${ }^{21,22}$ y poder generar una innovación en salud.

\section{Innovación en salud}

El concepto de innovación puede entenderse como una nueva forma de hacer las cosas, una mejora en algún producto, servicio o proceso. La visión más reciente lo plantea como un proceso complejo, multidireccional y sistémico que fluye desde la investigación a la aplicación, e involucra varios actores: investigadores, empresas, usuarios, etc. ${ }^{23}$. Según la OCDE, la innovación en salud es un proceso interactivo, circular, iterativo e interconectado, que esencialmente comprende cinco etapas: 1) la identificación de una necesidad; 2) la Investigación y el Desarrollo (I+D); 3) la comercialización; 4) el acceso, y 5) la difusión ${ }^{24}$. Los agentes claves en los procesos de innovación son diversos: actores políticos, autoridad sanitaria, pagadores, proveedores de servicios de la esfera pública y privada, y consumidores o pacientes ${ }^{25}$. A modo de ejemplo, y específicamente en el ámbito farmacológico, la innovación puede ser entendida como una nueva entidad molecular aprobada por la FDA, excluyendo los compuestos cosméticos ${ }^{26}$.

La innovación en salud es más compleja que en otros sectores productivos, ya que hay elementos sensibles que aumentan la resistencia entre los 
actores a la puesta en marcha y escalamiento de nuevas tecnología o procesos. Por ejemplo, los riesgos éticos y sociales de nuevos tratamientos, las reformas políticas, el marco regulatorio y, la inercia de quienes podrían adoptar la innovación ${ }^{27}$. En un sentido amplio, y bajo una mirada comercial, hay dos tipos de innovaciones en salud. La primera, no disruptiva, consiste en tratar de mejorar un producto o servicio disponible en el mercado actual. El segundo tipo, es lo que se conoce como innovación disruptiva o radicall. En ésta, existe un cambio total, un nuevo escenario, el producto o servicio genera nuevos mercado ${ }^{28}$, y hay creación de valor para quienes adoptan e implementan la innovación de manera exitosa ${ }^{1}$.

La innovación en el área de la salud puede no sólo mejorar la calidad del producto o servicio, sino que también hacerlo más accesible a la población. Hay tres formas en que la innovación en salud puede clasificarse ${ }^{29}$. La primera, es aquella innovación focalizada en el consumidor (o paciente); por ejemplo, mejoras en el tiempo de espera para obtener una hora médica, la calidad de servicio y atención, la conexión entre especialidades. La segunda, tiene que ver concretamente con la tecnología; nuevas drogas, métodos de diagnóstico, procedimientos, equipos médicos. El tercer aspecto, involucra nuevos modelos de negocios que impacten las organizaciones y su integración vertical y horizontal ${ }^{29}$.

La decisión de implementar una innovación en salud, por individuo u organización, de un producto o proceso ${ }^{30}$, varía según la perspectiva a considerar $^{31}$. Se han destacado las siguientes tres perspectivas:

\section{1) Perspectiva de la innovación}

Ésta se enfoca en las características de la innovación, y se puede resumir en cinco atributos principales: a) el avance relativo en comparación a lo existente; b) la compatibilidad con las actuales prácticas y hábitos (en torno a la implementación de la innovación); c) el grado de complejidad, que sea fácil de aplicar o usar; d) que sea fácil de "testear", antes de tomar una decisión final; y e) observación, si los resultados de la innovación son visibles para quienes los adoptan ${ }^{31}$.

\section{2) Perspectiva de quién adopta la innovación en salud: individuo u organización}

Una institución de salud puede tomar la decisión de comprar nuevos dispositivos médicos, sin embargo, son los médicos o profesionales paramédicos quienes deciden cómo y cuánto uso le darán. El grado de apertura para innovar en ideas y adoptarlas en organizaciones del sector salud, está basado en tres factores claves $^{32}$ : las características de la nueva tecnología, las particularidades de cada institución (como la cultura organizacional) y el entorno de mercado. Para que la innovación sea exitosa, ésta debe ser una prioridad estratégica en la organización ${ }^{33}$. En este sentido, destaca la Clínica Cleveland y el Centro Médico de la Universidad de Pittsburgh, quienes entregaron apoyo visible a la innovación en salud, generaron políticas de incentivos económicos para los investigadores, y pusieron énfasis en acelerar la adopción de nuevas tecnologías ${ }^{33}$. Por otro lado, también está el paciente y su mayor involucramiento mediante movilizaciones sociales vinculadas a salud; como es el caso del activismo que motivó la ley Ricarte Soto $^{34}$.

\section{3) Perspectiva del sistema de salud y su contexto}

Comprende las estructuras específicas en las cuales se enmarca el sistema de salud y la relevancia que tienen para la adopción y difusión en cada país; por ejemplo, el marco regulatorio para evaluar la seguridad de una nueva tecnología. La OCDE reconoce que los avances en biomedicina implican nuevos desafíos a nivel de políticas públicas. Para ello, sugiere que los gobiernos consideren las siguientes áreas temáticas de trabajo futuro: 1) mejorar la administración y el sistema regulatorio; 2) estudiar modelos de innovación abierta en salud; 3) comercializar la innovación derivada de investigación; 4) administrar adecuadamente los activos intelectuales (como patentes); 5) considerar las necesidades y demandas de los pacientes; 6) generar métricas e indicadores para la innovación en salud; y 7) encarar los desafíos de las tecnologías disruptivas ${ }^{24}$.

Por su complejidad, el proceso de innovar en salud requiere el análisis de la interdependencia de todos los actores relevantes en el proceso ${ }^{35-37}$; tanto a nivel de individuo, como de organización. A su vez, para la toma de decisiones y la administración en el área, es necesario comprender el concepto de medicina traslacional en el marco de los distintos componentes que permiten que un resultado científico consiga llegar a un producto, servicio o aplicación, y con ello impactar la salud del paciente (Figura 2). Adicionalmente, la me- 


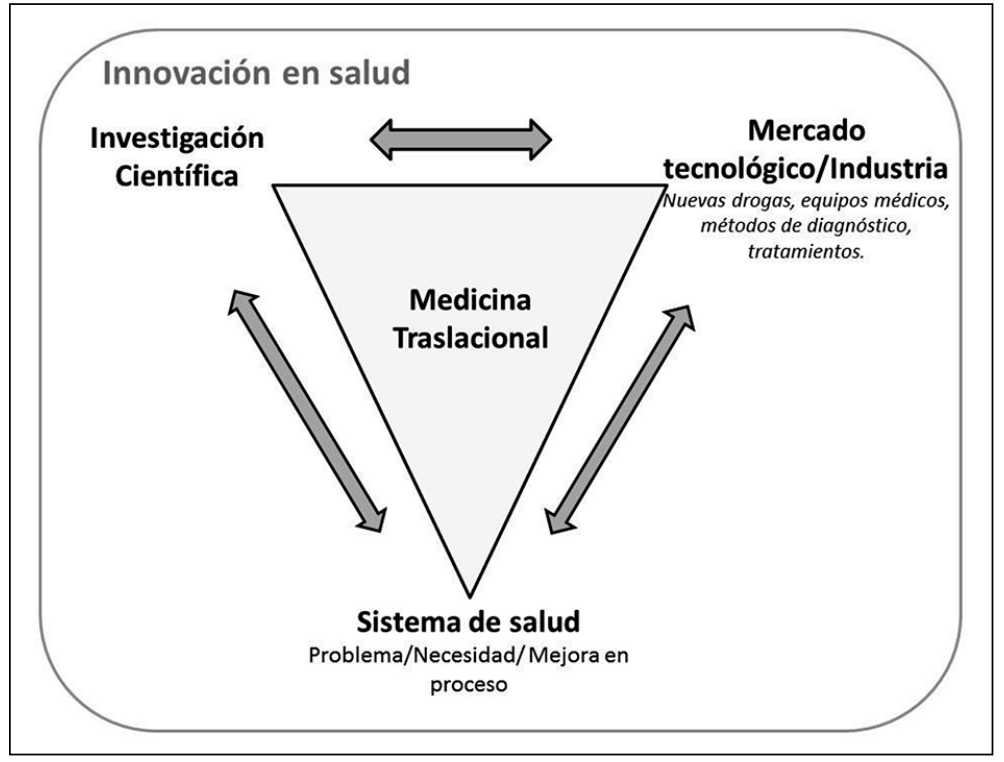

Figura 2. La innovación en salud es posible cuando hay comunicación entre los actores involucrados. Se destaca que no es un proceso lineal, sino de una serie de retroalimentaciones que permiten modificar y avanzar para que el producto, servicio, o aplicación médica pueda ser implementado satisfactoriamente y contribuir a solucionar un problema a nivel de individuo u organización sanitaria. Modelo de innovación en salud, elaborado a partir de Consoli \& Mina, 2009. dicina traslacional como proceso, debiera incluir el traspaso de las preocupaciones o necesidades de los pacientes y la población en general, a la comunidad científica $^{38}$. Lo anterior, enriquece las investigaciones y posibilita la pesquisa de puntos críticos que podrían acelerar el avance de una innovación y la incorporación de ésta al sistema de salud.

Considerando las definiciones y los conceptos revisados, se identifican las características que permiten contextualizar la innovación en el sector biomédico nacional.

\section{I+D y el mercado biotecnológico en Chile}

\section{i) Investigación en Chile}

Mediante instrumentos provenientes de CONICYT y de la Corporación de Fomento de la Producción (CORFO), el Estado ha buscado fortalecer la I+D en el país. Durante el 2014 se invirtieron US\$833 millones aproximadamente, lo que representa $0,38 \%$ del PIB nacional ${ }^{39}$; cifra por debajo del promedio de los países OCDE. El mayor porcentaje de ese gasto (39\%) fue ejecutado por Instituciones de Educación Superior (Figura 3 ), señalando a las universidades como actores claves en el sistema de innovación nacional. El año 2014, el monto total para todos los proyectos adjudicados en la disciplina "Ciencias Médicas y de la Salud" financiados por CONICYT, sumó US\$ 20,5 millones; 8,2\% en relación al total del financiamiento comprometido por la Institución para el mismo período ${ }^{40}$. La Tabla 2, resume los

\section{GASTO I+D EN CHILE AÑO 2014 US\$ 833 MILLONES (0,38\% DEL P.I.B.)}

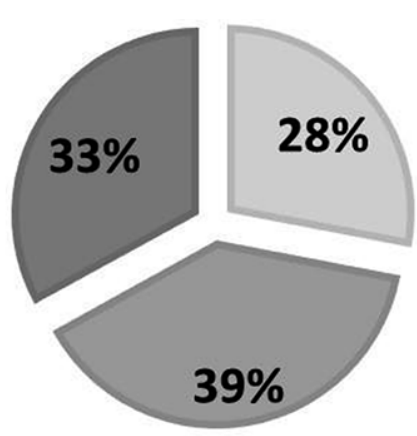

- Gasto I+D ejecutado por otros sectores

a Gasto I+D ejecutado por el sector Educación Superior n Gasto I+D ejecutado por empresas

Figura 3. Gasto I+D en Chile, año 2014. 
Tabla 2. Montos programas FONDECYT y FONDEF año 2014 y 2015

\begin{tabular}{|c|c|c|c|c|}
\hline \multirow[t]{2}{*}{ Programa CONICYT } & \multicolumn{2}{|c|}{$\begin{array}{l}\text { Monto comprometido } \\
\text { (MM\$) }\end{array}$} & \multicolumn{2}{|c|}{$\begin{array}{l}\text { Monto "Ciencias Médicas y Salud" } \\
\text { (MM\$) }\end{array}$} \\
\hline & Año 2014 & Año 2015 & Año 2014 & Año 2015 \\
\hline FONDECYT & 114.116 & 121.144 & 9.814 & 14.032 \\
\hline FONDEF & 12.101 & 16.453 & 1.580 & 2.162 \\
\hline
\end{tabular}

Elaboración propia con datos de CONICYT.

montos de los programas del "Fondo de Investigación Científica y Tecnológica" (FONDECYT) y del "Fondo de Fomento al Desarrollo Científico y Tecnológico" (FONDEF) durante los años 2014 y 2015.

Por su parte, el Fondo de Financiamiento de Centros de Excelencia en Investigación en Áreas Prioritarias (FONDAP) financia el fortalecimiento o creación de centros enfocados en problemas de gran importancia para el país en sectores claves ${ }^{40}$. De los trece centros FONDAP vigentes, dos abordan problemas de salud de forma directa: el Centro de Estudios Avanzados de Enfermedades Crónicas (ACCDIS, por sus siglas en inglés) y el Centro de Gerociencia, Salud Mental y Metabolismo ${ }^{41}$. Ambos centros, son patrocinados por la Pontificia Universidad Católica de Chile y la Universidad de Chile y cuentan con un presupuesto por cinco años de US\$ 6,7 millones cada uno. ACCDIS, fue adjudicado el año 2013 y su énfasis está puesto en las enfermedades cardiovasculares y cáncer ${ }^{42}$. GERO, fue adjudicado el año 2015, y mediante proyectos de investigación básica y clínica, pretende estudiar el impacto del envejecimiento en las enfermedades del cerebro, a nivel celular y molecular ${ }^{41}$.

Los hallazgos científicos realizados en Chile, mayoritariamente, presentan una oferta tecnológica con tendencia al "technology push", donde la innovación es "empujada" desde la ciencia hacia el mercado; a diferencia de su opuesto, el "market pull”, en que la tecnología se enfoca en una necesidad de la industria o al desarrollo de un nuevo mercado $^{43}$. Este aspecto condiciona gran parte de la transferencia de los resultados de investigación al sector productivo.

\section{ii) Mercado biotecnológico}

La biotecnología es el concepto que agrupa las nuevas tecnologías en genómica, proteómica, ingeniería de tejidos y terapia celular, que permiten avances en sectores como la salud humana, veterinaria, agricultura, procesamiento industrial y otras áreas de aplicación ${ }^{44}$. Según los indicadores de biotecnología de la OCDE, Estados Unidos, España y Francia son los países que concentran la mayor cantidad de empresas biotecnológicas; con $11.554,2.742$ y 1.950 compañías, respectivamente ${ }^{45}$. En Chile, la industria biotecnológica está en aumento, pero aún no es comparable con las economías desarrolladas. El año 2004 se fundó la Asociación Chilena de Biotecnología, ASEMBIO, con la finalidad de potenciar el crecimiento y cooperación entre las empresas del sector; en la actualidad, cuenta con más de 60 socios activos. Por su parte, la Agencia de Promoción de la Inversión Extranjera del Gobierno de Chile ${ }^{46}$, en una publicación reciente, señaló que el sector biotecnológico está compuesto por 100 instituciones, 79 privados y 22 consorcios; las ventas anuales se estiman en US\$900 millones. Cabe mencionar que $30 \%$ de estas empresas biotecnológicas pertenecen al ámbito de la salud, y dentro de las principales oportunidades de inversión en biomedicina, se destaca el desarrollo de vacunas y kits diagnóstico para cáncer, ensayos clínicos y desarrollo farmacéutico $^{46,47}$.

\section{Estrategias para la transferencia de los resultados de investigación desde las universidades a la industria}

Las universidades tienen un rol preponderante en la ejecución del gasto I+D en Chile. En este contexto, la escasa relación academia-industria se ha destacado como un factor esencial para poder transferir los conocimientos generados en las universidades ${ }^{43}$. La relación entre estos dos actores posee elementos que supeditan este vínculo: 1) la 
confianza; 2) la intensidad de las conexiones, la frecuencia con la que interactúan y se comunican; y 3 ) las distancias, tanto física, de conocimiento, o cultural ${ }^{48}$. Según cifras del Ministerio de Economía, Fomento y Turismo (2015), sólo 9,4\% de las grandes empresas colaboran en innovación con instituciones universitarias ${ }^{49}$. Esto ejemplifica uno de los grandes desafíos en la temática, aumentar los lazos entre la academia y el sector productivo, con el objeto de estimular la comunicación y la eventual transferencia de conocimientos.

Dentro las estrategias impulsadas por el Estado para disminuir las brechas entre ciencia y empresa, destacan: 1) Ley 20.241 de Incentivo tributario a la I+D, modificada el 2012, que permite rebajar en impuestos de primera categoría 35\% de los recursos destinados a actividades $\mathrm{I}+\mathrm{D}^{43}$; 2) Programas impulsados por CONICYT y CORFO para fortalecer las capacidades $\mathrm{I}+\mathrm{D}$ del país; 3) Fortalecimiento de Oficinas de Transferencia y Licenciamiento (OTL) al interior de universidades, con la misión de identificar, recolectar resultados de investigación y promover la investigación orientada a resolver problemáticas del sector productivo ${ }^{43}$; 4) Incentivos a la formación de "Hubs de Transferencia Tecnológica", para aumentar la proyección internacional de los negocios científico-tecnológicos y mejorar la gestión de la propiedad intelectual ${ }^{43}$.

\section{Conclusiones}

La innovación en salud es un tema contingente, cuyo interés aumenta con la divulgación de los resultados de investigación biomédica, la necesidad de mejorar la salud de los pacientes y el interés por disminuir los indicadores de morbilidad y mortalidad para patologías recurrentes y crónicas. En este sentido, el espectro del concepto de medicina traslacional y el involucramiento e interconexión de diversos actores (como investigadores, universidades, empresas, profesionales de la salud y sector público) son esenciales para el avance de una tecnología o aplicación que surge en el laboratorio de una universidad. En Chile, durante los últimos años, se han incrementado las estrategias públicas que estimulan la transferencia de los hallazgos científicos a la industria. Para evidenciar el impacto, es fundamental que los actores se relacionen y comuniquen, incentivando lazos de confianza que contribuyan a dinamizar el proceso de innovación en el área y acelerar la obtención de productos y/o servicios derivados de la investigación biomédica.

\section{Referencias}

1. Omachonu VK, Einspruch NG. Innovation in healthcare delivery systems: a conceptual framework. The Innovation Journal: The Public Sector Innovation Journal 2010; 15 (1): 1-20.

2. Gehr S, Garner CC. Rescuing the Lost in Translation. Cell 2016; 165 (4): 765-70.

3. Macleod MR, Michie S, Roberts I, Dirnagl U, Chalmers I, Ioannidis JP, et al. Biomedical research: increasing value, reducing waste. The Lancet 2014; 383 (9912): 101-4.

4. Ogilvie D, Craig P, Griffin S, Macintyre S, Wareham NJ. A translational framework for public health research. BMC public health 2009; 9 (1): 116.

5. Núñez P, Torres A, Armas R. ¿Se sustentan las Guías GES en trabajos científicos financiados por los fondos de CONICYT? Rev Med Chile 2014; 142 (12): 1540-6.

6. Jiang F, Zhang J, Wang X, Shen X. Important steps to improve translation from medical research to health policy. Journal of translational medicine 2013; 11 (1): 33.

7. Callard F, Rose D, Wykes T. Close to the bench as well as at the bedside: involving service users in all phases of translational research. Health Expectations 2012; 15 (4): 389-400.

8. van der Laan AL, Boenink M. Beyond bench and bedside: disentangling the concept of translational research. Health care analysis 2015; 23 (1): 32-49.

9. Wagner J, Kroetz D. Transforming Translation: Impact of Clinical and Translational Sciences. Clinical and translational science. 2016.

10. Tageja N. Bridging the translation gap-new hopes, new challenges. Fundamental \& clinical pharmacology. 2011; 25 (2): 163-71.

11. Grimshaw JM, Eccles MP, Lavis JN, Hill SJ, Squires JE. Knowledge translation of research findings. Implementation science 2012; 7 (1): 50.

12. Yao Q, Lyu P-H, Ma F-C, Yao L, Zhang S-J. Global informetric perspective studies on translational medical research. BMC medical informatics and decision making 2013; 13 (1): 77.

13. Fort DG, Herr TM, Shaw PL, Gutzman KE, Starren JB. Mapping the evolving definitions of translational research. Journal of Clinical and Translational Science 2017; 1 (1): 60-6. 
14. Sung NS, Crowley Jr WF, Genel M, Salber P, Sandy L, Sherwood LM, et al. Central challenges facing the national clinical research enterprise. JAMA 2003; 289 (10): 1278-87.

15. Trochim W, Kane C, Graham MJ, Pincus HA. Evaluating translational research: a process marker model. Clinical and translational science 2011; 4 (3): 153-62.

16. Harvard Catalyst. Clinical and Translational Research Spectrum, 2017. Disponible en: https://catalyst.harvard. edu/pathfinder/ [Consultado el 18 de enero de 2017].

17. Minsal, Instituto de Salud Pública de Chile, Departamento Agencia Nacional de Medicamentos. Ensayos Clínicos. Disponible en: http://www.ispch.cl/ensayos-clinicos [Consultado el 12 de junio de 2017].

18. Fernández-Moure JS. Lost in translation: the gap in scientific advancements and clinical application. Frontiers in Bioengineering and Biotechnology. 2016; 4.

19. Hanney SR, Castle-Clarke S, Grant J, Guthrie S, Henshall C, Mestre-Ferrandiz J, et al. How long does biomedical research take? Studying the time taken between biomedical and health research and its translation into products, policy, and practice. Health research policy and systems 2015; 13 (1): 1.

20. Morris ZS, Wooding S, Grant J. The answer is 17 years, what is the question: understanding time lags in translational research. Journal of the Royal Society of Medicine 2011; 104 (12): 510-20.

21. Roberts SF, Fischhoff MA, Sakowski SA, Feldman EL. Perspective: transforming science into medicine: how clinician-scientists can build bridges across research's "valley of death". Academic Medicine 2012; 87 (3): 26670.

22. Meslin EM, Blasimme A, Cambon-Thomsen A. Mapping the translational science policy 'valley of death'. Clinical and translational medicine 2013; 2 (1): 14.

23. Navarro JC, Benavente JM, Crespi G. The new imperative of innovation: policy perspectives for Latin America and the Caribbean. 2016.

24. OECD. Innovation Strategy, Biomedicine and Health Innovation. Synthesis Report. 2010. Disponible en: http://oecd.org/health/biotech/46925602.pdf [Consultado el 18 de enero de 2017].

25. Windrum P, García-Goni M. A neo-Schumpeterian model of health services innovation. Research Policy 2008; 37 (4): 649-72.

26. Munos B. Lessons from 60 years of pharmaceutical innovation. Nature reviews Drug discovery 2009; 8 (12): 959-68.

27. Länsisalmi H, Kivimäki M, Aalto P, Ruoranen R. Innovation in healthcare: a systematic review of recent research. Nursing science quarterly 2006; 19 (1): 66-72.
28. Price CP, John AS. Innovation in healthcare. The challenge for laboratory medicine. Clinica Chimica Acta 2014; 427: 71-8.

29. Herzlinger RE. Why innovation in health care is so hard. Harvard business review 2006; 84 (5): 58.

30. Reinhardt R, Hietschold N, Spyridonidis D. Adoption and diffusion of innovations in health care. Challenges and opportunities in health care management: Springer; 2015. p. 211-21.

31. Rogers EM. Diffusion of innovations/Everett M. Rogers. NY: Simon and Schuster, 2003-576 p. 2003.

32. Thakur R, Hsu SH, Fontenot G. Innovation in healthcare: Issues and future trends. Journal of Business Research 2012; 65 (4): 562-9.

33. Bates DW, Sheikh A, Asch DA. Innovative Environments In Health Care: Where And How New Approaches To Care Are Succeeding. Health Affairs 2017; 36 (3): 400-7.

34. Medina S, Kottow M. Ética de la protección y ley Ricarte Soto: de heridas y parches. Revista Chilena de Salud Pública 2015; 19 (3): 305-12.

35. Mittra J. The New Health Bioeconomy: R\&D Policy and Innovation for the Twenty-first Century: Springer; 2015.

36. Polese F, Capunzo M. The determinants of translational medicine success-A managerial contribution. Translational Medicine@UniSa 2013; 6: 29.

37. Consoli D, Mina A. An evolutionary perspective on health innovation systems. Journal of Evolutionary Economics 2009; 19 (2): 297.

38. Kon AA. The Clinical and Translational Science Award (CTSA) Consortium and the translational research model. The American Journal of Bioethics 2008; 8 (3): 58-60.

39. Ministerio de Economía Fomento y Turismo. Estudios y Encuestas: Quinta Encuesta Nacional sobre Gasto y Personal en Investigación y Desarrollo (año de referencia 2014), 2015. Disponible en: http://economia.gob.cl/ wp-content/uploads/2016/01/Presentaci\%C3\%B3n-resultados-I-D-2015.pdf [Consultado el 12 de marzo de 2017].

40. Ministerio de Educación, CONICYT, Gobierno de Chile. Compendio Estadístico Concursos CONICYT 2013-2015, 2016.

41. GERO. Centro de Gerociencia, Salud Mental y Metabolismo. Disponible en: http://www.gerochile.org [Consultado el 12 de marzo de 2017].

42. Ministerio de Educación, CONICYT, Centros FONDAP. Centro de Estudios de Enfermedades Crónicas (ACCDIS). Disponible en: http://www.conicyt.cl/fondap/ centros-fondap/accdis/ [Consultado el 12 de marzo de 2017]. 
43. Ministerio de Economía Fomento y Turismo, División de Innovación. Estudio cualitativo sobre el estado actual de la Transferencia Tecnológica en Chile, Informe Final, 2016. Disponible en: http://www.economia.gob. cl/wp-content/uploads/2016/07/Estudio-cualitativo-TT-en-Chile-1.pdf [Consultado el 12 de marzo de 2017].

44. Ebers M, Powell WW. Biotechnology: Its origins, organization, and outputs. North-Holland; 2007.

45. OECD. Innovation in science, technology and industry. Number of firms active in biotechnology, 2014. Disponible en: http://oecd.org/sti/biotech/keybiotechnologyindicators.htm [Consultado el 12 de marzo de 2017].

46. CIE CHILE Comité de Inversiones Extranjeras, Agencia de promoción de inversión extranjera de Chile. Opportunities for Biotechnology, 2015. Disponible en: https://www.elanbiz.org/documents/20182/100309/ Invest + in + Chile + Opportunities+for+Biotechnology/ cdad4919-30be-4efd-86d2-f40da57475a0?version=1.0 . [Consultado el 18 de enero de 2017].

47. DGE Consulting. Docencia Gestión Empresarial Investigaciones Económicas y Financieras. Estudio de Oportunidades de Inversión en el Sector de la Biotecnología en Chile, Informe Final para CIE CHILE, 2013.

48. Battistella C, De Toni AF, Pillon R. Inter-organisational technology/knowledge transfer: a framework from critical literature review. The Journal of Technology Transfer 2016; 41 (5): 1195-234.

49. Ministerio de Economía Fomento y Turismo, División de Innovación. Plan Nacional de Innovación 2014-2018, 2015. Disponible en: http://www.economia.gob.cl/ wp-content/uploads/2014/12/Plan-Nacional-de-Innovaci\%C3\%B3n1.pdf [Consultado en junio de 2017]. 